\title{
Identificación de consumo de alcohol por medio de la guía OMS/GI-mhGAP en el triaje de urgencias médicas
}

\author{
Identification of alcohol consumption with the WHO/GI-mhGAP guide in medical \\ emergency room triage
}

\author{
Viridiana G. Eslava-Torres ${ }^{1 *}$, Guadalupe X. Ortega-Ramírez ${ }^{1}$, Brenda A. Barrera-Flores ${ }^{1}$, \\ Ixchel Calixto-Molina ${ }^{1}$, Alma H. Guerrero-Martínez ${ }^{1}$, Lesly P. Díaz-Vásquez ${ }^{1}$, Katya F. Cabañas-Moreno ${ }^{1}$, \\ César De Lucio-Ramírez¹, Wendy V. Bernal-Ruíz ${ }^{1}$ y Edgar Landa-Ramírez ${ }^{1,2}$
}

${ }^{1}$ Departamento de Psicología Urgencias, División de Urgencias y Observación, Hospital General Dr. Manuel Gea González; ${ }^{2}$ Departamento de Psicología, Facultad de Psicología, Universidad Nacional Autónoma de México. Ciudad de México, México

\section{Introducción}

La Secretaría de Salud ${ }^{1}$ señaló que en 2017 se abordaron 657,127 pacientes dentro de triaje del servicio de urgencias, de los cuales 3706 fueron atendidos por uso de sustancias psicoactivas, como alcohol ${ }^{2}$, debido a que las consecuencias ponen en riesgo su vida o su función ${ }^{3,4}$. Por lo anterior, es relevante identificar el consumo riesgoso de alcohol en los pacientes que llegan al área de triaje de urgencias. La Organización Mundial de la Salud (OMS) recomienda el uso de la Guía de Intervención del Programa de Acción Global de Salud Mental (GI-mhGAP) versión 2.0, la cual permite identificar problemas neurológicos, adictivos y de salud mental, además de permitir la posibilidad de generar una intervención in situ que promueva el cambio de los problemas evaluados ${ }^{5}$.

El objetivo de este estudio fue adaptar la Gl-mhGAP 2.0 para el consumo de alcohol en pacientes en el triaje de urgencias médicas de un hospital general de la Ciudad de México.

\section{Método}

Estudio no experimental, analítico-observacional. Los participantes fueron 175 pacientes de triaje seleccionados por conveniencia; en su mayoría, los participantes eran hombres $(57 \%)$, con un promedio de edad de 38 años (desviación estándar [DE]: 15.36).

Se utilizaron cuatro instrumentos:

- Cuestionario de datos sociodemográficos y datos médicos creado ex profeso.

- Adaptación de la GI-mhGAP 2.0 para pacientes de triaje de urgencias médicas conformada por tres preguntas filtro, de las que cada una indica el nivel de riesgo de consumo, además del tipo de intervención que se debería realizar.

- Prueba de detección de consumo de alcohol, tabaco y sustancias (ASSIST, Alcohol, Smoking and Substance Involvement Screening Test) ${ }^{6}$, con propiedades psicométricas en la población.

- Cuestionario de identificación de los trastornos debidos al consumo de alcohol (AUDIT, Alcohol Use Disorders Identification Test) ${ }^{7}$, con propiedades psicométricas en la población.

Las evaluaciones se realizaron durante el periodo conocido como "Guadalupe-Reyes" en el espacio de triaje mientras los pacientes esperaban su turno; el personal de psicología se acercaba a invitarles a la investigación, les daba a conocer el objetivo y recababa su consentimiento informado de forma oral. Una vez

\section{Correspondencia:}

*Viridiana G. Eslava-Torres

E-mail: viridiana.eslava.8@gmail.com
Disponible en internet: 07-03-2022

Fecha de recepción: 30-11-2021

Fecha de aceptación: 16-12-2021 DOI: 10.24875/REIE.21000213
Rev Educ Investig Emer. 2022;4(Supl 1):32-34 www.medicinadeemergencias.com bajo la licencia CC BY-NC-ND (http://creativecommons.org/licenses/by-nc-nd/4.0/). 


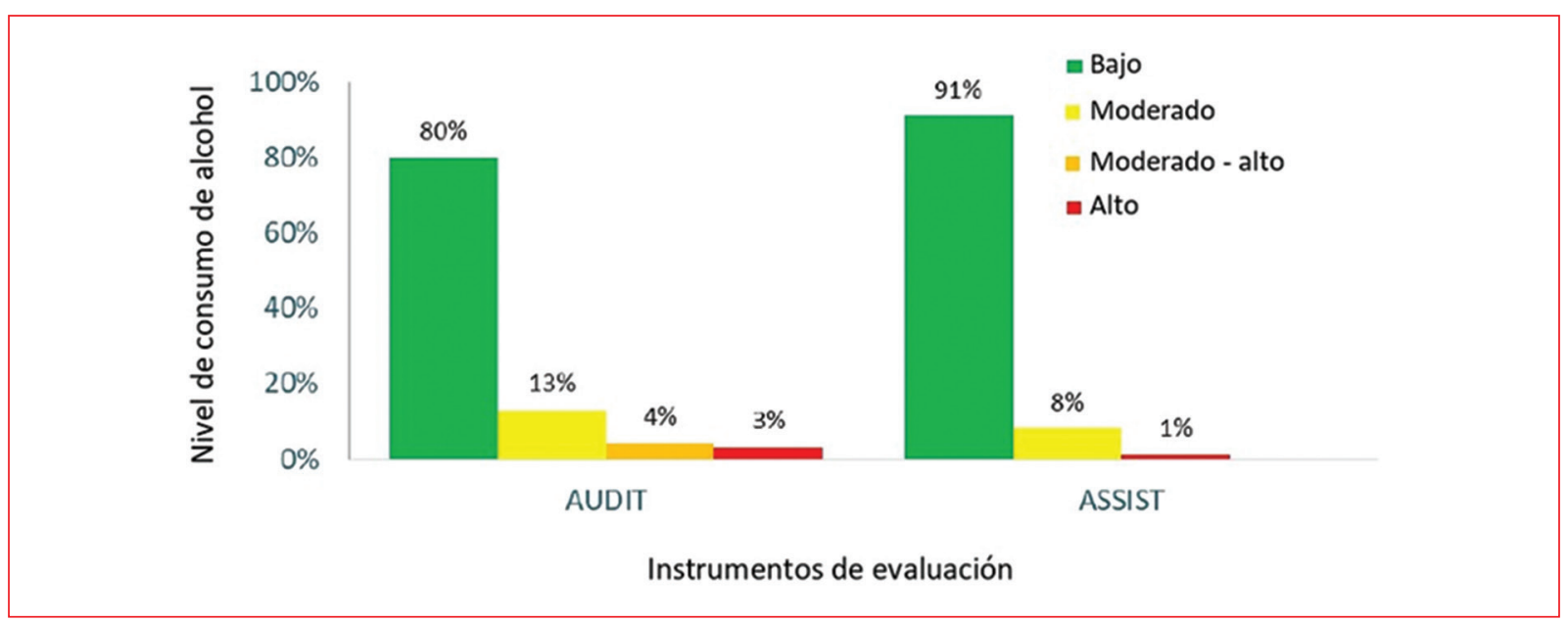

Figura 1. Nivel de consumo de alcohol según la prueba de detección de consumo de alcohol, tabaco y sustancias (ASSIST) y el cuestionario de Identificación de los trastornos debidos al consumo de alcohol (AUDIT) en usuarios de triage de urgencias médicas.

que el paciente aceptaba participar, se le preguntaban datos sociodemográficos y datos médicos, y después se aplicaba el algoritmo de consumo de sustancias incluyendo los dos instrumentos antes mencionados. Finalmente, se daba intervención de acuerdo con lo indicado por la Gl-mhGAP 2.0 según el nivel de consumo, además de brindar el contacto de centros que atienden el consumo de sustancias. Por otro lado, al médico que atendió a la persona evaluada se le preguntó: «¿ldentificó consumo de sustancias (alcohol) en el paciente?».

Para el análisis de datos se hicieron análisis descriptivos, así como el coeficiente Kappa de Cohen; se utilizó el programa R Studio 3.6.1.

\section{Resultados}

Las evaluaciones y las intervenciones se realizaron en un tiempo promedio de 15.8 minutos (DE: 7.61). Del total de las personas evaluadas, el $98 \%$ completaron exitosamente el algoritmo de consumo de sustancias. Con respecto a las puntuaciones de AUDIT y ASSIST, hay concordancia en que la mayoría de la población presenta un consumo bajo $(80 \%$ y $91 \%$, respectivamente), mientras que una minoría mantiene un consumo elevado o riesgoso ( $3 \%$ y $1 \%$, respectivamente) (Fig. 1).

Los datos de la percepción del médico con respecto al consumo de sustancias se completaron en un $46 \%$ y se obtuvo un nivel de concordancia bajo, estadísticamente significativo ( $k=0.326 ; p=0.003$ ), entre las percepciones del médico y del psicólogo.

\section{Conclusiones}

De acuerdo con el objetivo propuesto en este estudio y los datos obtenidos, el consumo de bebidas alcohólicas está presente en los usuarios de triaje de urgencias médicas; partiendo de esto, la adaptación de la GI-mhGAP 2.0 parece ser una opción viable para identificarlos e intervenir.

Esta investigación tuvo algunas limitaciones, como el tamaño de la muestra y la validación del algoritmo. Sin embargo, muestra algunas bondades, como la disminución de la brecha de atención en salud mental presente en contextos dinámicos, urgentes y variables como lo es el triaje, así como el permitir prevenir, detectar y brindar intervención sobre el consumo de alcohol. Este trabajo se suma a la poca evidencia que existe en la literatura sobre el tema.

Es necesario continuar realizando investigación para corroborar los datos obtenidos en este estudio.

\section{Agradecimientos}

Agradecemos a todo el equipo de urgencias médicas del Hospital General Dr. Manuel Gea González por su apoyo constante para con el equipo de psicología, pues brindaron las condiciones necesarias para que este trabajo fuera posible. Agradecemos también a nuestros compañeros del equipo de psicología de Urgencias, por su apoyo incondicional, trabajo en equipo y calidez humana. Finalmente, gracias a todos los participantes de esta investigación por compartir su experiencia con nosotros. 


\section{Financiamiento}

Este proyecto no tiene relación económica y se encuentra fuera de cualquier tipo de patrocinio 0 financiamiento.

\section{Conflicto de intereses}

Los autores declaran no tener ningún conflicto de intereses.

\section{Responsabilidades éticas}

Protección de personas y animales. Los autores declaran que para esta investigación no se han realizado experimentos en seres humanos ni en animales.

Confidencialidad de los datos. Los autores declaran que han seguido los protocolos de su centro de trabajo sobre la publicación de datos de pacientes.

Derecho a la privacidad y consentimiento informado. Los autores han obtenido el consentimiento informado de los pacientes y/o sujetos referidos en el artículo. Este documento obra en poder del autor de correspondencia.

\section{Bibliografía}

1. Secretaría de Salud de la Ciudad de México. Agenda Estadística 2017. 2016. Disponible en: http://data.salud.cdmx.gob.mx/portal/media/agenda 2017/urgencias.html

2. Secretaría de Salud. Sistema de Vigilancia Epidemiológica en Adicciones. Informe SISVEA 2016. 2015. Disponible en: https://epidemiologia. salud.gob.mx/gobmx/salud/documentos/info_sisvea/informes_sisvea_2016.pdf

3. Organización Mundial de la Salud. Informe sobre el estado mundial del alcohol y la salud. 2014. Disponible en: https://apps.who.int/iris/bitstream/ handle/10665/112736/9789240692763_eng.pdf;jsessionid=2AB4C3E2DABA3C80FE0A088FAC68AEC5? sequence $=1$

4. American College of Emergency Physicians. Definition of Emergency Medicine. 2021. Disponible en: https://www.acep.org/patient-care/policy-statements/definition-of-emergency-medicine/

5. Organización Mundial de la Salud. La prueba de detección de consumo de alcohol, tabaco y sustancias (ASSIST). Manual para uso de atención primaria. 2011. Disponible en: https://apps.who.int/iris/bitstream/handle/10665/85403/9789275332368_spa.pdf?sequence=1\&isAllowed=y

6. Organización Mundial de la Salud. AUDIT: Cuestionario de Identificación de los Trastornos debidos al Consumo de Alcohol. 2001. Disponible en: https:// www.who.int/substance_abuse/activities/en/AUDITmanualSpanish.pdf

7. Organización Mundial de la Salud. Guía de intervención mhGAP para los trastornos mentales, neurológicos y por consumo de sustancias en el nivel de atención de salud no especializada. Versión 2-0. 2016. Disponible en: https://iris.paho.org/handle/10665.2/34071 